\title{
Combined $\beta$-Adrenergic and Cholinergic Antagonism Produces Behavioral and Cognitive Impairments in the Water Maze: Implications for Alzheimer Disease and Pharmacotherapy with $\beta$-Adrenergic Antagonists
}

\author{
Andrea J Saber ${ }^{1,2}$ and Donald P Cain*,I \\ 'Department of Psychology, Graduate Program in Neuroscience, University of Western Ontario, London, Ontario, Canada
}

\begin{abstract}
This study examined the effects of $\beta$-adrenergic and muscarinic blockade on spatial learning and strategy use in the water maze. Male Long-Evans rats received systemic injections of propranolol (PRO; 10 or $20 \mathrm{mg} / \mathrm{kg}$ ) or scopolamine (SCO; 0.3 or $1.0 \mathrm{mg} / \mathrm{kg}$ ) either singly or in combination. To separate strategies learning from spatial learning approximately half of the rats underwent water maze strategies pretraining prior to drug administration and spatial training. PRO did not impair performance in any group. SCO impaired naive but not pretrained rats. $\mathrm{PRO}$ and $\mathrm{SCO}$ given together in high doses impaired all aspects of behavior in both naive and pretrained rats, and caused sensorimotor disturbances in some groups. PRO $(10 \mathrm{mg} / \mathrm{kg})$ and $\mathrm{SCO}(0.3 \mathrm{mg} / \mathrm{kg})$ together caused a specific spatial reversal learning impairment in pretrained rats without causing strategies impairments or sensorimotor disturbances. Nadolol administered with SCO failed to produce the same impairments as PRO, suggesting that PRO produced its effects by acting on central nervous system sites. These results point to a greater than additive impairing effect of PRO and SCO on adaptive behavior, and a specific role for $\beta$-adrenergic and cholinergic systems working in conjunction in spatial learning. They also suggest that some of the behavioral and cognitive impairments seen in Alzheimer patients or patients receiving pharmacotherapy with $\beta$-adrenergic antagonists in which cholinergic activity is also compromised may result from the combined impairment of $\beta$-adrenergic and cholinergic systems.

Neuropsychopharmacology (2003) 28, 1247-1256, advance online publication, 9 April 2003; doi: I0.1038/sj.npp. I 300163
\end{abstract}

Keywords: learning; memory; scopolamine; propranolol; acetylcholine; noradrenaline; alzheimers

\section{INTRODUCTION}

A variety of different drug treatments impair performance by rats in the water maze task. These include treatments that antagonize muscarinic, NMDA, or AMPA receptors or that serve as GABA agonists by binding to the benzodiazepine receptor (Bannerman et al, 1995; Beiko et al, 1997a; Cain, 1997, 1998; Cain et al, 1996, 1997, 2000; Morris et al, 1986; Morris, 1989; Saucier and Cain, 1995; Saucier et al, 1996; Vanderwolf, 1987; Whishaw, 1989; Whishaw and Tomie, 1987). Certain other treatments, such as depletion of serotonin by $p$-chlorophenylalanine (PCPA), antagonism of $\beta$-adrenergic receptors with propranolol (PRO), or destruc-

\footnotetext{
*Correspondence: Dr DP Cain, Department of Psychology, University of Western Ontario, London, Ontario, Canada N6A 5C2, Tel: + I 519 661 2111 84628, Fax: + 519661 3961, E-mail: cain@uwo.ca

${ }^{2}$ Current address: Department of Psychology, Dalhousie University, Halifax, Nova Scotia, Canada B3H 4JI.

Received 30 September 2002; revised 12 December 2002; accepted 17 December 2002

Online publication: 06 January 2003 at http://www.acnp.org/citations/ Npp0I0603450
}

tion of noradrenalin-containing neurons in the locus coeruleus, have little effect or no on water maze performance (Beiko et al, 1997a; Decker et al, 1990; Vanderwolf and Baker, 1996). In contrast to treatment with a single drug, combined treatment with two drugs can markedly impair water maze performance even if treatment with either drug alone causes little or no impairment. Thus rats given a low dose of either PRO or scopolamine (SCO), a muscarinic cholinergic antagonist, had search times comparable to saline controls, whereas a combination of the two treatments significantly increased search times (Decker et al, 1990). Similarly, rats given PCPA had no water maze impairment, and rats given only SCO had a moderate impairment, whereas rats given both treatments were severely impaired (Beiko et al, 1997a). The findings from these experiments are important because they reveal a greater than additive impairing effect on behavior compared to the effect of each drug alone. This suggests that interactions between neurotransmitter systems may be very significant for the production of adaptive behavior. The findings also suggest a link between the behavioral and cognitive impairments that occurs in Alzheimer disease and 
the fact that both noradrenergic and cholinergic functions are frequently impaired in Alzheimer patients (Bowen and Francis, 1990; Hardy et al, 1985; but see also Haroutunian et al, 1990). They may also be relevant to pharmacotherapy with both noradrenergic and cholinergic antagonists, which together can produce behavioral and cognitive impairments (McAinsh and Cruickshank, 1990; Miller, 1995; Rogers and Bowman, 1990). Further study of combined noradrenergic and cholinergic antagonism seems warranted.

The most obvious manifestation of the water maze impairments discussed above is an increase in search time to find the hidden platform (e.g., Morris et al, 1986; Sutherland et al, 1982). Although an increase in search time is typically interpreted as a spatial learning impairment, very little published research has studied the possibility that increased search times can result from impairments in functions other than spatial learning. The water maze task requires that a naive rat habituate to the testing situation and acquire a variety of skills and strategies for coping with and obtaining information in the task before a specific spatial location can be learned (Morris, 1989; Whishaw, 1989). The required water maze skills and strategies include swimming, searching away from the pool wall (where the platform is never located) by suppressing a natural inclination to swim thigmotaxically, recognizing that the hidden platform is the only refuge, and using the hidden platform as refuge (Cain, 1998). Once these skills and strategies are acquired, information about the platform location in relation to distal spatial cues can be obtained, allowing the rat to learn the specific location of the hidden platform in the pool. Thus, success in the spatial component of the task is dependent on prior acquisition of the required skills and strategies.

Studies indicate that in many cases drug treatments impair acquisition and use of required water maze strategies by naive rats (Bannerman et al, 1995; Beiko et al, 1997a; Cain, 1997, 1998; Cain et al, 1996, 1997, 2000; Saucier et al, 1996), thus obscuring any conclusion on whether the drug specifically impairs spatial learning. This problem could be avoided if the strategy learning and spatial learning components of the task could be separated. This is possible using a strategies pretraining procedure (hereafter, pretraining; Morris, 1989). During pretraining thick black curtains surround the pool to occlude distal visual cues in the water maze test environment, and the hidden platform is moved to a new location after each trial. Thus, the rat does not learn a specific platform location during pretraining. Instead, it learns the strategies that are required in the task and also becomes habituated to the handling and test environment. The latter point is important given that the water maze task is highly stressful, as documented by a rapid and large surge in serum corticosterone early in training (Beiko et al, 1997b). The use of pretraining together with a detailed behavioral analysis has shown that strategy impairments and sensorimotor disturbances, but not spatial learning impairments, are the main cause of increased search times in naive animals given SCO (Beiko et al, 1997a; Cain et al, 2000; Saucier et al, 1996), or the benzodiazepine diazepam (Cain, 1997; Cain et al, 2000), or any of a variety of NMDA receptor antagonists (Bannerman et al, 1995; Cain et al, 1996, 1997, 2000; Hoh et al, 1999; Saucier and Cain, 1995). In each of the above studies pretrained drugged rats were indistinguishable from controls on behavioral measures of spatial learning. The fact that stress is known to impair performance on a variety of behavioral tasks, and that pretraining both reduces blood levels of corticosterone and eliminates behavioral impairments during subsequent water maze spatial training, suggests that a component of the pretraining effect may result from the reduction in the stress response in pretrained rats (Beiko et al, 1997b; Holscher, 1999).

The present study further examined the effects of single and combined administration of PRO and SCO on water maze acquisition. As pretraining and a detailed behavioral analysis were not previously employed in the earlier water maze study with PRO and SCO (Decker et al, 1990), a goal of the present study was to better understand the roles of $\beta$ adrenergic and muscarinic systems in strategy acquisition and use, spatial learning, and sensorimotor function by using pretraining and a detailed behavioral analysis. Another goal was to obtain dose-response information on the effect of PRO and SCO on behavior by including higher doses of PRO or SCO than were used previously (Decker et al, 1990). To understand how the drugs affected sensorimotor function outside the maze, a beam task (Kolb and Whishaw, 1985) was used to assess sensorimotor ability.

\section{MATERIALS AND METHODS}

\section{Subjects}

Male Long-Evans rats $(N=152$, Charles River, Canada) weighing 300-450 g were used. The rats were housed in pairs on a $12: 12 \mathrm{~h}$ light-dark cycle (lights on at $7 \mathrm{am}$ ) with testing during the light phase. Food and water were available ad libitum. Prior to testing all animals were removed from their cages and periodically handled. All animal procedures were in accordance with the Guide for the Care and Use of Experimental Animals of the Canadian Council on Animal Care.

\section{Drug Administration}

Drug treatment groups are presented in Table 1. A criterion for drug dose selection was that the treatments do not interfere with swimming and climbing behavior in the water maze. PRO ((S)-(-)-propranolol hydrochloride, Sigma) was given in a dose of 10 or $20 \mathrm{mg} / \mathrm{kg}$, in a volume of $1.0 \mathrm{ml} / \mathrm{kg}$ administered intraperitoneally. PRO readily crosses the blood-brain barrier and antagonizes both $\beta-1$ and $\beta-2$ noradrenergic receptors in the thalamus, neocortex, and hippocampal complex (Booze et al, 1989). A $10 \mathrm{mg} / \mathrm{kg}$ dose was used because this dose is sufficient to block noradrenergically induced increases in the activity of hippocampal neurons (Harley and Sara, 1992) without affecting water maze search time in naive rats (Decker et al, 1990) or spontaneous locomotor and exploratory behavior in rats (Sara et al, 1995). A dose of $20 \mathrm{mg} / \mathrm{kg}$ was also used because this dose increases immobility and decreases rearing in the open field (Angrini et al, 1998). To evaluate the possibility that peripheral effects of PRO could be a factor in water maze performance nadolol (NAD; a hydrophilic $\beta$-adrenergic antagonist that does not cross the blood-brain barrier 
Table I Groups and Treatments

\begin{tabular}{|c|c|c|c|}
\hline & Treatment & Dose/amount & $n$ \\
\hline \multicolumn{4}{|l|}{ Low dose groups } \\
\hline Naive SCO.3 & Scopolamine & $0.3 \mathrm{mg} / \mathrm{kg}$ & 8 \\
\hline Pretrained SCO.3 & Scopolamine & $0.3 \mathrm{mg} / \mathrm{kg}$ & 8 \\
\hline Naive PROIO & Propranolol & $10 \mathrm{mg} / \mathrm{kg}$ & 8 \\
\hline Pretrained PROIO & Propranolol & $10 \mathrm{mg} / \mathrm{kg}$ & 8 \\
\hline Naive PRO I0+SCO.3 & Propranolol+scopolamine & $10 \mathrm{mg} / \mathrm{kg}, 0.3 \mathrm{mg} / \mathrm{kg}$ & 8 \\
\hline Pretrained PRO I0+SCO.3 & Propranolol+scopolamine & $10 \mathrm{mg} / \mathrm{kg}, 0.3 \mathrm{mg} / \mathrm{kg}$ & 8 \\
\hline \multicolumn{4}{|l|}{ High dose groups } \\
\hline Naive SCOI & Scopolamine & $1.0 \mathrm{mg} / \mathrm{kg}$ & 9 \\
\hline Pretrained SCOI & Scopolamine & $1.0 \mathrm{mg} / \mathrm{kg}$ & 8 \\
\hline Naive PRO20 & Propranolol & $20 \mathrm{mg} / \mathrm{kg}$ & 8 \\
\hline Pretrained PRO20 & Propranolol & 20 mg/kg & 8 \\
\hline Naive PROI0+SCOI & Propranolol+scopolamine & $10 \mathrm{mg} / \mathrm{kg}, 1.0 \mathrm{mg} / \mathrm{kg}$ & 11 \\
\hline Pretrained PROI0+SCOI & Propranolol+scopolamine & $10 \mathrm{mg} / \mathrm{kg}, 1.0 \mathrm{mg} / \mathrm{kg}$ & 8 \\
\hline Naive PRO20+SCOI & Propranolol+scopolamine & $20 \mathrm{mg} / \mathrm{kg}, 1.0 \mathrm{mg} / \mathrm{kg}$ & 8 \\
\hline Pretrained PRO20+SCOI & Propranolol+scopolamine & $20 \mathrm{mg} / \mathrm{kg}, 1.0 \mathrm{mg} / \mathrm{kg}$ & 12 \\
\hline Naive NAD5+SCOI & Nadolol+scopolamine & $5.0 \mathrm{mg} / \mathrm{kg}, 1.0 \mathrm{mg} / \mathrm{kg}$ & 12 \\
\hline Pretrained NAD5+SCOI & Nadolol+scopolamine & $5.0 \mathrm{mg} / \mathrm{kg}, 1.0 \mathrm{mg} / \mathrm{kg}$ & 8 \\
\hline \multicolumn{4}{|l|}{ Control groups } \\
\hline Naive control & Saline+saline & $1.0 \mathrm{ml} / \mathrm{kg}, 1.0 \mathrm{ml} / \mathrm{kg}$ & 6 \\
\hline Pretrained control & Saline+saline & $1.0 \mathrm{ml} / \mathrm{kg}, 1.0 \mathrm{ml} / \mathrm{kg}$ & 6 \\
\hline
\end{tabular}

Naive and pretrained control groups did not differ on any measure and were combined (see text). $n=$ number in group; $\mathrm{NAD}=$ Nadolol; $\mathrm{PRO}=$ propranolol; $\mathrm{SCO}=$ scopolamine.

effectively, Schiff and Saxey, 1984) was administered to certain groups (see Table 1). A $5.0 \mathrm{mg} / \mathrm{kg}$ dose was chosen because this dose increased fecal bolus production in the absence of catalepsy in rats (Sachdev and Sabaror, 1997).

SCO was given in doses of 0.3 or $1.0 \mathrm{mg} / \mathrm{kg}$ in a volume of $1.0 \mathrm{ml} / \mathrm{kg}$, administered intraperitoneally. A $0.3 \mathrm{mg} / \mathrm{kg}$ dose was chosen because this dose did not affect water maze search times in naive rats (Decker et al, 1990). A $1.0 \mathrm{mg} / \mathrm{kg}$ dose was chosen because this dose elevates water maze search times in naive but not pretrained rats (Saucier et al, 1996). A methylated muscarinic antagonist was not used because neither atropine methylnitrate nor methylscopolamine affect search times in the water maze (Hagan et al, 1986).

Animals treated with both PRO and SCO concurrently received two separate injections. Therefore, control rats were given two individual saline injections $(1.0 \mathrm{ml} / \mathrm{kg}$, intraperitoneal). At $20 \mathrm{~min}$ prior to all testing rats were given injections of $\operatorname{drug}(\mathrm{s})$, or saline, as indicated in Table 1.

\section{Apparatus}

Water maze. The water maze was a white cylindrical pool (diameter $1.5 \mathrm{~m}$ ) filled with water $($ depth $60 \mathrm{~cm}$ ) that was made opaque by the addition of floating white polypropylene pellets (Cain et al, 1993). Approximately $2 \mathrm{~cm}$ below the surface was a $15 \times 15 \mathrm{~cm}^{2}$ hidden platform. There were numerous distal cues in the testing room (cupboards, doors, posters on the walls, objects hanging from the ceiling). A video camera that was recessed into the ceiling above the pool sent feed to a video recorder and to an automated digital tracking system (PolyTrak, San Diego Instruments) to acquire swim path data.
Beam task. A narrow wooden beam $(1.8 \mathrm{~cm}$ wide and $86 \mathrm{~cm}$ long), located $1 \mathrm{~m}$ from the ground, was used to measure sensorimotor coordination. A practice beam $(6 \mathrm{~cm}$ wide and $86 \mathrm{~cm}$ long) was first used to habituate rats to the procedure. A halogen quartz lamp $(1000 \mathrm{~W})$ was positioned at the start of the beam. At the end of the beam was a dark goal area $\left(50 \times 50 \mathrm{~cm}^{2}\right)$ that was covered with woodchip bedding. Apart from the halogen lamp the room was unilluminated. On the floor below the beam was a bin of woodchips, providing a soft surface if the rats fell.

\section{Testing Procedures and Behavioral Analysis}

Strategies pretraining. Pretraining was performed according to Morris (1989), and trained the rats in the required task skills and strategies without providing them with spatial information to swim to a specific platform location. Approximately half of the animals in the study underwent pretraining prior to spatial training, including control rats (see Table 1). During pretraining the pool was surrounded by a thick black curtain that hung from a track attached to the ceiling and completely surrounded the pool. The curtain was uniform and provided no directional cues. The rats received three trials a day ( $4 \mathrm{~h}$ intertrial interval) for 4 days (12 trials total) with the release points randomized across trials. Each rat was placed in the pool facing the wall from a pseudorandomized cardinal starting position on each trial. The rat swam until it found and mounted the platform or until $120 \mathrm{~s}$ had elapsed. If it did not find the platform by $120 \mathrm{~s}$ it was guided there by the experimenter, where it remained for $15 \mathrm{~s}$. The hidden platform was moved to a different quadrant of the pool on successive trials for each rat, thus preventing the learning of a place response. No drugs were given before or during pretraining. After each 
trial the rats were kept in a holding cage under a heat lamp until dry to avoid a loss in body core temperature.

Spatial training. Spatial training began on the fifth day after the completion of pretraining, if given (Morris, 1989). The black curtain was removed, allowing the rats to make full use of the distal cues in the room to guide navigation. Rats were tested in squads of four or five and were given drug or control treatments $20 \mathrm{~min}$ prior to the start of training as indicated in Table 1. Each rat was given 10 trials to find the hidden platform ( 5 min intertrial interval), with the platform in the center of the southeast quadrant. Rats were introduced into the water adjacent to and facing the pool wall at the north, south, east, or west, and swam for a maximum of $60 \mathrm{~s}$ or until they found and climbed onto the platform. If a rat did not find the platform during a trial, it was manually guided there, where it remained for $15 \mathrm{~s}$. The order of release points was pseudorandomized subject to the constraint that summed distances from the start points to the platform in each two-trial block were approximately equal across blocks. The experimenter monitored each trial on a television screen and was not visible to the rats during the trials. Rats were placed under a heat lamp between trials to avoid loss in body core temperature.

Day 2 of testing followed the same protocol except that the platform was located in the northwest quadrant, providing reversal training.

Water maze acquisition measures. Digital files of trials were objectively analyzed for two measures of task acquisition, hidden platform search time, and direct and circle swims. Hidden platform search time was the time from release into the pool until the rat mounted the platform. The criterion for a direct swim was that the rat remain within an $18 \mathrm{~cm}$-wide virtual 'alley' from the start point to the platform (Whishaw and Tomie, 1987). A circle swim was a swim trajectory that approximated an arc of a circle from the start point to the platform without exceeding $360^{\circ}$ of circling or crossing over itself (Whishaw and Jarrard, 1995). Direct and circle swims were analyzed because they describe the swim paths that normal rats take in this task (Cain, unpublished data), and because they are the most stringent measure of spatial memory available for this task (Whishaw and Jarrard, 1995; Whishaw et al, 1995).

Behavioral strategies measures. Use of water maze strategies was evaluated with two measures. Swim time in the pool periphery was obtained by analyzing the digitized swim paths using PolyTrak for swim time in the outer $50 \%$ of the pool area, within $20 \mathrm{~cm}$ of the pool wall, where the platform was never located at any time during the study. This was performed because swimming away from the wall to search the inner region of the pool is an essential strategy in this task, and has a major impact on the search time measure of acquisition (Morris, 1989; Schenk and Morris, 1985). Various pharmacological treatments and brain lesions markedly impair acquisition of this strategy by naive rats (Beiko et al, 1997a; Cain, 1998; Cain et al, 1996).

The second strategies measure was use of the hidden platform as a refuge. This was quantified by scoring the videotapes for deflections and swimovers, which constituted platform contact errors (Beiko et al, 1997a; Cain, 1998; Cain et al, 1996). A deflection occurred when a rat bumped into the hidden platform during a trial and continued swimming without mounting the platform. A swimover occurred if a rat climbed onto the hidden platform and immediately swam off the other side in a continuous motion. Illustrations of these platform contact errors have been published (Cain, 1998). Deflections and swimovers constitute strategy errors and increase search time. Summed deflections and swimovers as a percentage of the total number of contacts with the hidden platform during spatial training provided a measure of platform contact errors.

Swimming ability was assessed by measuring swim speed as $\mathrm{cm} / \mathrm{sec}$ during a free swim in the pool after the completion of spatial training.

Beam task. Sensorimotor function outside the maze was assessed using the beam task (Kolb and Whishaw, 1985). Previous studies (Beiko et al, 1997a; Cain et al, 2000) showed that water maze experience does not affect beam task performance. Therefore, a subset of the rats used in the water maze phase of the study was randomly selected for beam task testing. The rats were divided into one of the following groups (group notation similar to that in Table 1): PRO10 $(n=6) ; \operatorname{PRO} 20(n=5) ;$ SCO.3 $(n=5)$; SCO1 $(n=6)$; $\mathrm{PRO} 10+\mathrm{SCO} .3(n=4) ; \mathrm{PRO} 10+\mathrm{SCO} 1(n=7) ; \mathrm{PRO} 20+\mathrm{S}-$ CO1 $(n=4)$; Control $(n=4)$. Rats were first given 7-10 trials with a wide $(6 \mathrm{~cm})$ beam to habituate to the procedure. They were then given $7-10$ trials with the narrow $(1.8 \mathrm{~cm})$ beam to ensure that they would locomote to the end of the beam. The requisite drug/control treatment was then administered and beginning $20 \mathrm{~min}$ later rats were placed individually under the lamp at the start of the beam and allowed to traverse for a maximum of $60 \mathrm{~s}$. A total of 10 trials were given consecutively. A slip was defined as hanging from the beam by one or both forepaws. If the rat fell a default score of $60 \mathrm{~s}$ was assigned. The time taken to traverse the beam was recorded with a stopwatch.

\section{RESULTS}

\section{Strategies Pretraining}

Mean search times to find the hidden platform ranged from 35.2 to $60.1 \mathrm{~s}$ in various groups on day 1 of pretraining, and ranged from 11.1 to $24.8 \mathrm{~s}$ in the same groups on the last day of pretraining. The reduced search times and observations of behavior (swimming away from the wall; use of the hidden platform as a refuge) during pretraining are similar to results reported previously using the same technique (Morris, 1989), and indicate that the pretrained animals acquired and made use of the strategies necessary in the task.

\section{Behavioral Effects of Low Doses}

Data for naive and pretrained saline-treated rats were combined since they did not differ on any behavioral measure. These rats will be referred to as the control group. Repeated measures of ANOVA were used to analyze search and periphery time, and one-way ANOVA was used to analyze all other water maze measures. Post hoc comparisons were carried out using Dunnett's test. 


\section{Search and Periphery Time}

Search time to find the hidden platform on day 1 is shown in Figure 1a for naive low-dose groups and in Figure 1c for pretrained low-dose groups. Drug treatment resulted in longer search times in the naive SCO.3 group and the naive PRO10 + SCO. 3 group (Group: $F(6,53)=6.69, p<0.0001$; naive SCO.3 and naive PRO10+SCO.3 vs Control, $p<0.05$; Figure 1a). Search time decreased as training progressed (Trial: $\mathrm{F}(4,180)=63.70, p<0.0001$ ). Naive groups had longer search times than pretrained groups $(p<0.05)$. On day 2 , the naive $\mathrm{PRO} 10+\mathrm{SCO} .3$ group had longer search times than controls (Group: $\mathrm{F}(6,53)=2.39$, $p<0.05$; naive PRO10 + SCO.3 vs Control, $p<0.05$; data not shown).

Time spent in the periphery on day 1 is shown in Figure $1 \mathrm{~b}$ for naive groups and in Figure 1d for pretrained groups. Drug treatment led to increased periphery time in the naive SCO.3 and naive PRO10+SCO. 3 groups (Group: $\mathrm{F}(6,53)=6.17, p<0.0001$; nave SCO. 3 and nave PRO10 + SCO.3 vs Control, $p<0.05$; Figure $1 \mathrm{~b})$. Periphery swimming decreased as training progressed (Trial: $\mathrm{F}(3,158)=59.69$, $p<0.0001)$. On day 2 , no group differences were found ( $p>0.05$; data not shown).

\section{Direct and Circle Swims}

Summed direct and circle swims are shown as a percentage of all swims during spatial training on day 1 in Figure 2a. Drug treatment impaired direct and circle swims in the naive SCO. 3 and naive $\mathrm{PRO} 10+\mathrm{SCO} .3$ groups (Group: $\mathrm{F}(6,59)=5.13, \quad p<0.0001$; naive SCO.3 and naive PRO10 + SCO. 3 group $v s$ Control, $p<0.05$; Figure 2a). Naive groups had fewer direct and circle swims than pretrained groups $(p<0.05)$.

Summed direct and circle swims during reversal training on day 2 are presented in Figure $2 \mathrm{~b}$. On day 2 drug treatment impaired direct and circle swims in both the naive and pretrained PRO10+SCO.3 groups (Group: $\mathrm{F}(6,59)=4.21, p=0.002$; naive and pretrained PRO10+ SCO.3 vs Control, $p<0.05$; Figure $2 \mathrm{~b}$ ).

\section{Use of the Hidden Platform as a Refuge}

The incidence of platform contact errors on day 1 was $17.8 \pm 12.0 \%$ (mean + SEM) in the naive PRO10 + SCO.3 group and $1.8 \pm 1.2 \%$ in controls. Data for the other groups were comparable to controls. Analysis indicated a nonsignificant trend between groups $(\mathrm{F}(6,59)=1.97, p=0.086)$. By day 2 of spatial training all groups had learned to use the hidden platform as a refuge (data not shown).

\section{General Observations}

The mean swim speed did not differ between any of the groups ( $p>0.05$; data not shown). Digitized swim paths on the last trial of day 1 from the rat with the median total search time of its group during spatial training on day 1 are shown in Figure 3. The poor performance of the naive $\mathrm{PRO} 10+\mathrm{SCO} .3$ rat is consistent with the greater periphery swimming and longer search times found in this group. The good performance of the naive SCO.3 rat suggests that by the end of spatial training on day 1 this rat had learned something $t$ about water maze strategies and the hidden platform location despite the fact that this group had impairments on day 1 .
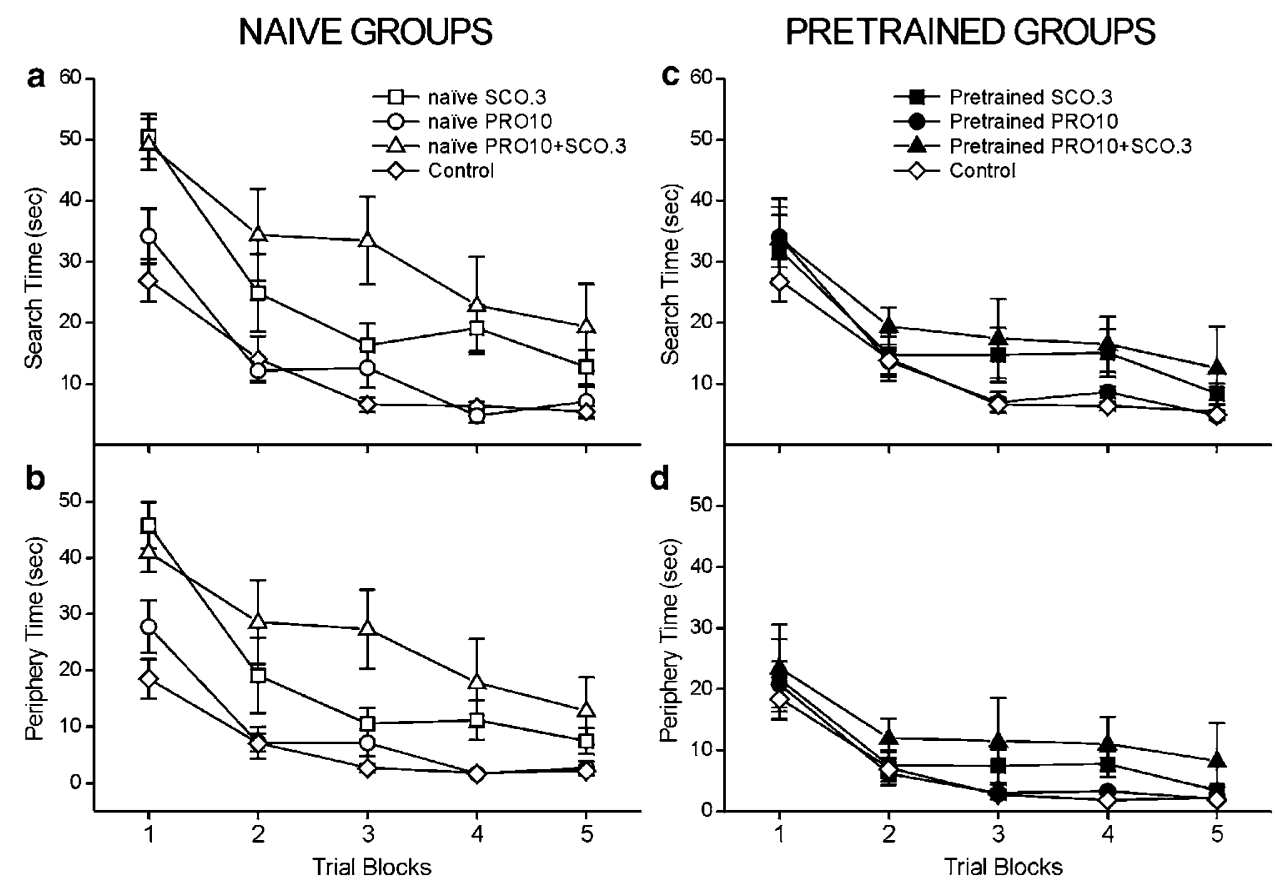

Figure I Hidden platform search time (a, c) and periphery swim time (b, d) on day I for low-dose groups. The data points represent the mean of two trials as trial blocks; for example, trial block $I$ is the mean search time of spatial training trials $I$ and 2 . The values in this and the following figures represent means + SEM. Search time and periphery time appear strongly related in both naive and pretrained groups because periphery swim time is a major component of search time. See text for definitions of measures and Table I for group designations. 
Direct and Circle Swims

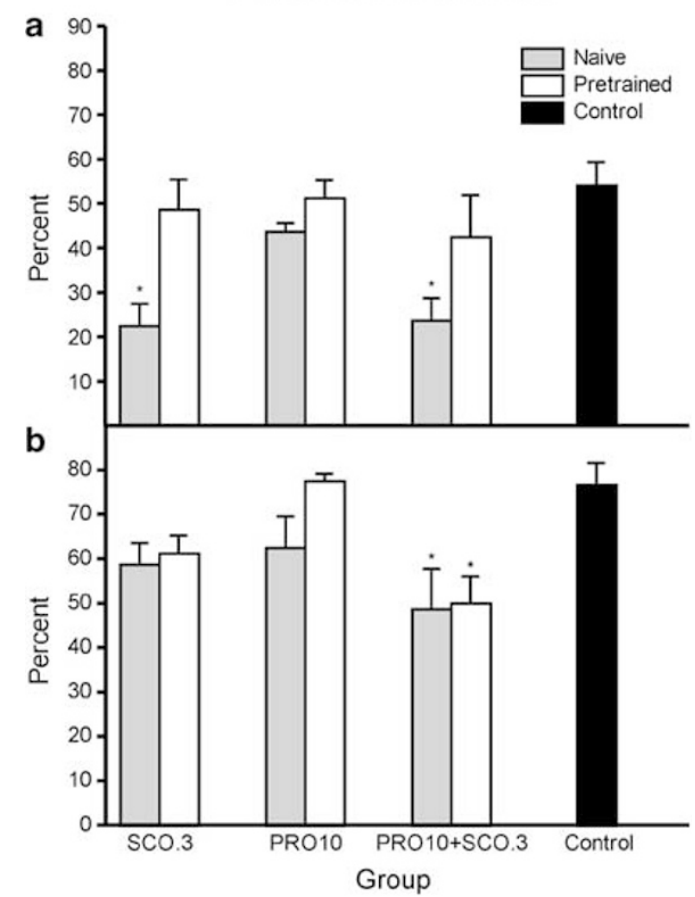

Figure 2 Percentage of direct and circle swims for low-dose groups as a percentage of all swims during training on day $\mid$ (a) and during reversal training on day 2 (b). The group labels along the $X$-axis of panel (b) apply to panel (a) also. The asterisk (*) indicates values that are significantly different from controls $(p<0.05)$.

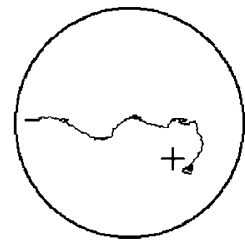

Naïve PRO10

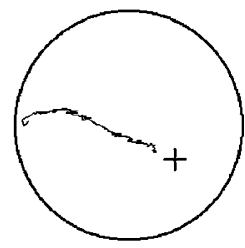

Pretrained PRO10

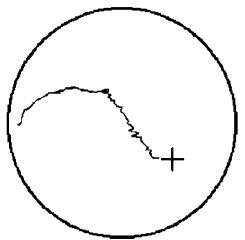

Naïve SCO.3

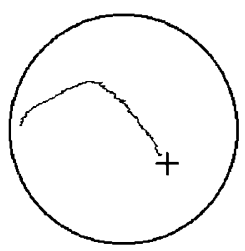

Pretrained SCO.3

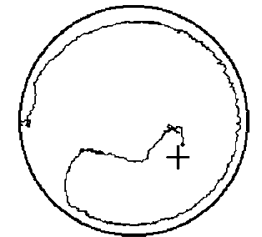

Naive PRO10+SC0.3

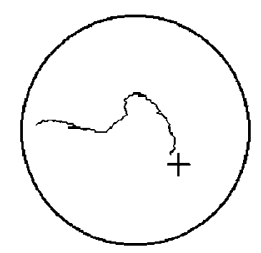

Pretrained PRO10+SCO.3
Figure 3 Digitized swim paths of the last training trial on day I for the rat in each low-dose group with the median total search time during spatial training on that day. The hidden platform is indicated by the cross $(+)$ in the lower right quadrant of the pool. The paths of the single-treatment rats indicate excellent performance by the end of day I of spatial training. The path of the naive PROIO+SCO.3 rat indicates excessive periphery swimming and the path of the pretrained $\mathrm{PRO} O \mathrm{O}+\mathrm{SCO} .3$ rat indicates swimming away from the hidden platform near the midpoint in the swim.

\section{Beam Task}

A one-way ANOVA was used to analyze time to traverse the beam and number of slips and falls. There were no group differences for either measure ( $p>0.05$; data not shown).

\section{Behavioral Effects of High Doses}

The naive SCO1 group was impaired on all water maze measures on day 1 and was impaired on most measures on day 2, whereas the pretrained SCO1 group was not different from controls on any water maze measure on either day (data not shown). The PRO20 groups were also not different from controls on any measure on either day (data not shown). When treatments were given in combination, impairments were found in all measures in both naive and pretrained groups (data not shown).

\section{Effects of NAD}

NAD had more limited effects on water maze performance than PRO when it was administered with SCO. The naive and pretrained NAD5 + SCO1 groups had longer search times and more periphery swimming than controls on day 1 $(p<0.05)$ but did not differ from controls on day $2(p>0.05$; data not shown). The naive NAD5 + SCO1 group had fewer direct and circle swims than controls on day $1(p<0.05)$ but did not differ from controls on day 2 ( $p>0.05$; data not shown). No other comparisons involving NAD-treated groups were significant, and the pretrained NAD5 + SCO1 group did not differ from controls on any measure on either day ( $p>0.05$; data not shown).

\section{General Observations}

The mean swim speed did not differ between any of the groups on either day ( $p>0.05$; data not shown). Representative digitized swim paths (as defined above) on day 1 are shown in Figure 4. The naive and pretrained PRO20 and the pretrained SCO1 groups displayed excellent spatial memory at the end of spatial training on day 1 . The other groups swam excessively in the periphery, especially rats of the naive $\mathrm{PRO} 20$ + SCO1 group, which seldom swam away from the wall during spatial training. However, the pretrained $\mathrm{PRO} 10+\mathrm{SCO} 1$ and the pretrained $\mathrm{PRO} 20+\mathrm{SCO} 1$ rats searched the inner region of the pool during part of the trial, indicating some degree of retention of appropriate search strategies acquired during pretraining.

\section{Beam Task}

Performance on the beam task generally paralleled results from the water maze measures. The groups treated with both PRO and SCO were impaired on one or both beam task measures and the other groups were marginally impaired or unimpaired (data not shown).

\section{Correlations}

Pearson's product moment correlations were calculated between search time, periphery swim time, and platform contact errors. Data from all groups were included in the correlations. The calculated coefficients all ranged between 


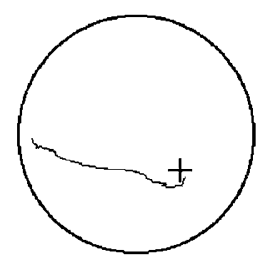

Naïve PRO20

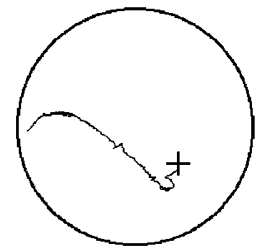

Pretrained PRO20

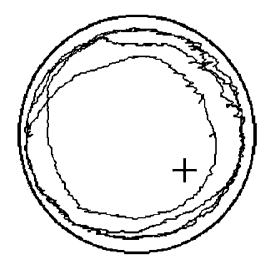

Naïve SCO1

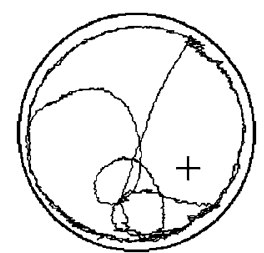

Naïve PRO10+SCO1

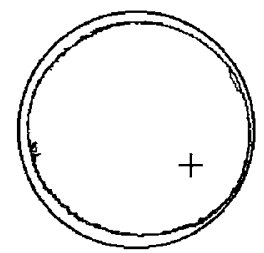

Naĩve PRO20+SCO1
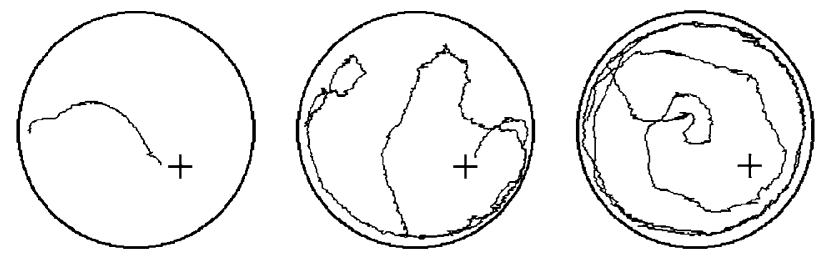

Pretrained SCO1 Pretrained PRO10+SCO1 Pretrained PRO20+SCO1

Figure 4 Digitized swim paths of the last training trial on day I for the rat in each high-dose group with the median total search time during spatial training on that day. The hidden platform is indicated by the cross (+). The naive PRO20, the pretrained PRO20, and the pretrained SCOI groups displayed excellent performance by the end of day I of spatial training. The other groups displayed poor performance, with excessive periphery swimming and little or no evidence of memory for the hidden platform location. Most of the rats that displayed poor performance failed to contact the hidden platform during the 60 s swim, and the naive PRO20 + SCOI rat failed to swim away from the wall at any time during the trial.

+0.41 and +0.98 , with associated $p$-values of $<0.001$ to $<0.0001$. The pattern of correlations indicated associations between impairments in strategy use and search time similar to those found previously in studies involving rats treated with neurotransmitter antagonists (Beiko et al, 1997a; Cain, 1997; Cain et al, 1996; Saucier et al, 1996).

\section{DISCUSSION}

Antagonism of $\beta$-adrenergic receptors alone did not impair any aspect of performance in either naive or pretrained rats. Antagonism of muscarinic receptors impaired performance by naive rats on day 1 , but pretrained rats were not impaired. The impairment we found in naive rats with $0.3 \mathrm{mg} / \mathrm{kg}$ SCO contrasts with the lack of an impairment with this dose in a previous study (Decker et al, 1990). This may be because this dose appears to be near the threshold for behavioral effects in naive rats (Cain et al, 2000; Vanderwolf, 1991). Apart from that finding, these results are consistent with previous research using these treatments (Beiko et al, 1997a; Cain et al, 2000; Decker et al, 1990; Saucier et al, 1996).

The most important results were obtained from groups given both antagonists. Naive rats given either low or high doses of PRO and SCO, and pretrained rats given high doses of PRO and SCO had impairments in both water maze strategies and spatial navigation. In addition, the correlations revealed significant associations between strategies impairments and increased search time, and there was evidence of sensorimotor impairment on the beam task in rats given high doses of PRO and SCO. Among these groups, there was no evidence of a selective spatial learning impairment as evaluated by the direct and circle swim measure. In each instance, impairment in this measure was accompanied by impairments in behavioral strategies that are crucial for obtaining information about the location of the hidden platform and for performing behaviors required to demonstrate knowledge of the hidden platform location. Results from control groups given both NAD and a high dose of SCO suggest that the effects of PRO resulted from actions on the central nervous system.

The results from the pretrained PRO10+SCO.3 group were of special interest. In contrast to the naive PRO10 + SCO.3 group, the pretrained PRO10+SCO.3 group never exhibited water maze strategy impairments on either day, indicating excellent retention and use of the strategies they acquired during pretraining. The pretrained $\mathrm{PRO} 10+\mathrm{SCO} .3$ group also displayed good spatial learning on day 1, with direct and circle swims that were comparable to controls. However, this group was impaired on the direct and circle swim measure on day 2 relative to controls. This impairment is similar to that seen in rats with hippocampal formation lesions, which can acquire an initial place response but cannot learn a place reversal (Whishaw and Jarrard, 1995, 1996; Whishaw et al, 1995). The present results indicate that combined antagonism of $\beta$-adrenergic and muscarinic receptors can produce a specific spatial reversal learning impairment in the absence of behavioral strategy or sensorimotor impairments.

\section{Relation to Previous Research}

The present results extend earlier work suggesting that a variety of different neurotransmitters is normally involved in the acquisition and performance of various components of the water maze task. While $\beta$-adrenergic mechanisms do not appear to be crucial for any component of the task, the fact that combined treatment with PRO and SCO caused water maze impairments that were greater than those caused by SCO alone suggests that $\beta$-adrenergic mechanisms normally contribute to these abilities. The nature of the impairments depended on both the past experience of the rats and the doses of PRO and SCO that were 
administered. Similar performance impairments were found with combined administration of SCO and PRO in rats tested in a three-panel runway task (Kobayashi et al, 1995).

Similar findings of a selective spatial learning impairment with drugs administered in combination have been obtained previously. For example, although treatment with either SCO or an NMDA receptor antagonist alone did not cause water maze impairments in pretrained rats (Bannerman et al, 1995; Cain et al, 2000; Saucier and Cain, 1995; Saucier et al, 1996), combined treatment with SCO and an NMDA receptor antagonist impaired spatial learning in pretrained rats without impairing water maze strategies (Cain et al, 2000). Similarly, treatment with diazepam did not cause water maze impairments in pretrained rats (Cain, 1997; Cain et al, 2000), whereas combined treatment with SCO and diazepam impaired spatial learning in pretrained rats without impairing water maze strategies (Cain et al, 2000).

More severe nonspecific behavioral impairments similar to those reported here with high doses of PRO and SCO administered together also have been reported previously. For example, although blockade of serotonin biosynthesis by PCPA did not impair acquisition of the water maze task by naive rats (Beiko et al, 1997a; Nilsson et al, 1988; Richter-Levin and Segal, 1989), combined treatment with PCPA and SCO produced a profound impairment of all adaptive behavior in the water maze and beam tasks, whether rats were naive or pretrained (Beiko et al, 1997a; Vanderwolf, 1987). The fact that PRO exhibits a limited degree of antiserotonergic acivity (Giarcovich and Enero, 1984) suggests that a factor in the impairments we found with combined administration of PRO and SCO may be the limited antiserotonergic effect of PRO.

Taken together, the previous and present findings suggest that among the systems studied to date there does not appear to be a crucial single system that is required for the spatial learning component of the conventional water maze task. Rather, the data suggest that a number of neurotransmitter systems normally contribute to spatial learning but that none of them, considered individually, is essential for this component. The specific nature of any impairments produced by the drugs appears to depend on both the past history of the animals, the specific actions of the drugs, and the doses that are administered.

\section{Basis of the Behavioral Impairments}

In studies of the kind reported here, the greatest behavioral impairments were found when serotonergic and cholinergic systems were antagonized simultaneously (Beiko et al, 1997a; Nilsson et al, 1988; Richter-Levin and Segal, 1989; Vanderwolf, 1987). Cholinergic cells in the basal forebrain and serotonergic cells in the brain stem provide an important form of ascending control over the electrical activity of the hippocampus and neocortex. Simultaneous disruption of both systems can completely abolish spontaneous forebrain activation, produce marked sensorimotor impairments, and impair a large variety of adaptive behaviors (Beiko et al, 1997a; Nilsson et al, 1988; RichterLevin and Segal, 1989; Vanderwolf, 1987). Thus, this combination of treatments appears to produce a general impairment of cerebral function and adaptive behavior. In contrast, selective disruption of either cholinergic or serotonergic inputs does not completely abolish spontaneous forebrain activation or spatial learning (Beiko et al, 1997a; Saucier et al, 1996; Vanderwolf, 1987; Vanderwolf and Baker, 1996). Similarly, selective disruption of noradrenergic inputs to the forebrain does not abolish spontaneous forebrain activation or spatial learning (Decker et al, 1990; Vanderwolf and Baker, 1996). Taken together the findings point to a direct role of serotonergic and cholinergic inputs in maintaining normal forebrain activation, but a somewhat lesser role of noradrenergic inputs in this function (Vanderwolf and Baker, 1996). This difference in the roles of these systems may be due in part to the quantitative differences in the number of each type of terminal in the neocortex. For example, serotonin terminals are approximately 10 times more numerous than noradrenergic terminals in the neocortex (Beaudet and Descarries, 1978; Vanderwolf and Baker, 1996). Nevertheless, all three systems project diffusely to most of the neocortex and appear to modulate cortical cellular function (McCormik, 1992). Antagonism of these different systems has effects on the behavior that depends on the system(s) that are antagonized and the degree of the antagonism.

\section{Conclusions and Implications}

Our use of pretraining allowed us to detect a specific impairment in spatial learning in the absence of strategies or sensorimotor impairments using appropriate doses of PRO and SCO. As discussed above, a similar outcome occurred in earlier studies with other combinations of treatments. Taken as a whole, these studies suggest that (1) a research design involving pretraining and a detailed behavioral analysis can distinguish treatment effects on spatial learning from effects on required behavioral strategies or sensorimotor function, (2) different combinations of treatments involving different actions in the central nervous system are capable of producing a specific impairment of spatial learning, and (3) a variety of different neurotransmitter systems and receptor populations normally contribute to spatial learning, but no single system or population appears to be crucial.

These results appear to have implications for research on Alzheimer disease. Noradrenergic and cholinergic function are frequently impaired in Alzheimer patients (Bowen and Francis, 1990; Hardy et al, 1985). These patients frequently exhibit maladaptive behaviors such as wandering away from home, inability to make use of landmarks in a familiar environment (Fairburn and Hope, 1988; Teri et al, 1988), and repetitive activities that appear purposeless (De Deyn et al, 1999). These symptoms appear to be similar to the spatial navigation learning impairment and repetitive thigmotaxic swimming behavior seen in the present study. The current findings may model some of the particularly maladaptive aspects of behavioral dysfunction in Alzheimer disease. Possible interactions between noradrenergic and cholinergic systems in producing normal electroencephalographic activity and behavior in normal brain, and impaired activity and behavior in Alzheimer brain have been discussed (Vanderwolf and Baker, 1996; Dringenberg, 2000).

In addition, our results may have implications for pharmacotherapy. Elderly patients with hypertension are 
often treated with PRO. Case studies report that such patients experience cognitive side effects including confusion and psychiatric disturbance (Rogers and Bowman, 1990). It is possible that some of these patients may be suffering from compromised cholinergic function because of manifest or developing Alzheimer disease. Treatment with a centrally acting $\beta$-adrenergic antagonist may potentiate their cognitive impairment. Further, some hypertensive patients may be given a $\beta$-adrenergic antagonist while taking anticholinergics for a variety of other illnesses. Drug classes with anticholinergic properties include antihistamines, antipsychotics, antidepressants, antispasmodics, and anti-Parkinsonian agents (Miller, 1995). The behavioral and cognitive effects of this polypharmacotherapy need to be better characterized. This can be carried out by studies of adaptive behaviors involving learning, memory, and sensorimotor function involving human patients. There is clinical evidence that the greater the lipophilicity of a $\beta$-adrenergic antagonist, the more profound its central side effects such as insomnia, nightmares, hallucinations, and memory loss (McAinsh and Cruickshank, 1990), and that peripheral $\beta$-adrenergic antagonism produces fewer cognitive side effects than PRO in hypertensive patients. Westerlund (1985) found that hypertensive patients given PRO had significantly more nightmares and hallucinations than individuals treated with atenolol, a hydrophilic $\beta$-adrenergic antagonist that does not readily cross the blood-brain barrier. Importantly, both drugs had the same effect on heart rate and blood pressure in the patients. This suggests comparable peripheral but differing central effects of the treatments. Treatment with a $\beta$-adrenergic antagonist that primarily acts in the periphery may be a more viable option for elderly patients who may be developing cholinergic dysfunction or for individuals currently taking anticholinergic treatmants. NAD is one such antagonist that acts on $\beta$-1 and 2-adrenergic receptors in the periphery with very little activity in the central nervous system. As shown here, when administered with SCO, NAD impaired adaptive behavior less than PRO. Further studies using a variety of doses of NAD with SCO will be needed to better clarify the effect of peripheral and central $\beta$-adrenergic antagonism on adaptive behavior.

\section{ACKNOWLEDGMENTS}

Supported by a grant to DPC and by a postgraduate scholarship to AS from the Natural Sciences and Engineering research Council of Canada.

\section{REFERENCES}

Angrini M, Leslie JC, Shephard RA (1998). Effects of propranolol, buspirone, pCPA, reserpine, and chlordiazepoxide on open-field behavior. Pharm Biochem Behav 59: 387-397.

Bannerman DM, Good MA, Butcher SP, Ramsay M, Morris RGM (1995). Distinct components of spatial learning revealed by prior training and NMDA receptor blockade. Nature 378: 182-186.

Beaudet A, Descarries L (1978). The monoamine innervation of rat cerebral cortex: synaptic and non-synaptic axon terminals. Neuroscience 3: 851-860.

Beiko J, Candusso L, Cain DP (1997a). The effect of nonspatial water maze pretraining in rats subjected to serotonin depletion and muscarinic receptor antagonism: a detailed behavioural assessment of spatial performance. Behav Brain Res 88: 201-211.

Beiko J, Lander R, Hampson E, Cain DP (1997b). An examination of the relationship between sex differences in spatial performance and plasma corticosterone levels in the rat. Abstr Canad Soc Brain Behav Cog Sci 8: 17.

Booze RM, Crisostomo EA, Davis JN (1989). Species differences in the localization and number of CNS beta-receptors: rat versus guinea pig. J Pharm Exp Ther 249: 911-920.

Bowen DM, Francis PT (1990). Neurochemistry, neuropharmacology and aetiological factors in Alzheimer's disease. Neuroscience 2: $101-108$.

Cain DP (1997). Prior non-spatial pretraining eliminates sensorimotor disturbance and impairments in water maze learning caused by diazepam. Psychopharmacology 130: 313-319.

Cain DP (1998). Testing the NMDA, long-term potentiation and cholinergic hypotheses of spatial learning. Neurosci Biobehav Rev 22: 181-193.

Cain DP, Inghanian K, Boon F (2000). Individual and combined manipulation of muscarinic, NMDA, and benzodiazepine receptor activity in the water maze task: implications for a rat model of Alzheimer dementia. Behav Brain Res 11: 125-137.

Cain DP, Saucier D, Boon F (1997). Testing hypotheses of spatial learning: the role of NMDA receptors and NMDA-mediated long-term potentiation. Behav Brain Res 84: 179-193.

Cain DP, Saucier D, Hall J, Hargreaves EL, Boon F (1996). Detailed behavioral analysis of water maze acquisition under APV or CNQX: contribution of sensorimotor disturbances to druginduced acquisition deficits. Behav Neurosci 110: 86-102.

Cain DP, Saucier D, Hargreaves EL, Wilson E, DeSouza JFX (1993). Polypropylene pellets as an inexpensive reusable substitute for milk powder in the Morris water maze. J Neurosci Methods 49: 193-197.

Decker MW, Gill MT, McGaugh JL (1990). Concurrent muscarinic and $\beta$-adrenergic blockade in rats impairs place-learning in a water maze and retention of inhibitory avoidance. Brain Res 513: 81-85.

DeDeyn PP, Rabheru K, Rasmussen A, Bocksberger JP, Dautzenberg PLJ, Eriksson S et al (1999). A randomized trial of risperidone, placebo, and haloperidol for behavioral symptoms of dementia. Neurology 53: 946-955.

Dringenberg HC (2000). Alzheimer's disease: more than a 'cholinergic disorder'-evidence that cholinergic-monoaminergic interactions contribute to EEG slowing and dementia. Behav Brain Res 115: 235-249.

Fairburn G, Hope RA (1988). Changes in behaviour in dementia: a neglected research area. Br J Psychiatry 152: 406-407.

Giarcovich S, Enero MA (1984). Decreased brain serotonergic activity after acure propranolol. Eur J Pharmacol 100: 123-125.

Hagan JJ, Tweedie F, Morris RGM (1986). Lack of task specificity and absence of posttraining effects of atropine on learning. Behav Neurosci 100: 483-493.

Hardy J, Adolfsson R, Alafuzoff I, Bucht G, Marcusson J, Nyberg P et al (1985). Transmitter deficits in Alzheimer's disease. Neurochem Int 7: 545-563.

Harley CW, Sara SJ (1992). Locus coeruleus bursts induced by glutamate trigger delayed perforant path spike amplitude potentiation in the dentate gyrus. Exp Brain Res 89: 581-587.

Haroutunian V, Santucci AC, Davis KIL (1990). Implications of multiple transmitter system lesions for cholinomimetic therapy in Alzheimer's disease. Prog Brain Res 84: 333-346.

Hoh T, Beiko J, Boon F, Cain DP (1999). Complex behavioral strategy and reversal learning in the water maze without NMDA receptor-dependent long-term potentiation. J Neurosci 19, RC2 $1-5$.

Holscher C (1999). Stress impairs performance in spatial water maze learning tasks. Behav Brain Res 100: 225-235. 
Kolb B, Whishaw IQ (1985). Earlier is not always better: behavioral dysfunction and abnormal cerebral morphogenesis following neonatal cortical lesions in the rat. Behav Brain Res 17: 25-43.

Kobayashi M, Ohno M, Yamamoto T, Watanabe S (1995). Concurrent blockade of $\mathrm{B}$-adrenergic and muscarinic receptors disrupts working memory but not reference memory in rats. Physiol Behav 58: 307-314.

McAinsh J, Cruickshank JM (1990). Blockers and central nervous system side effects. Pharmacol Ther 46: 163-197.

McCormick DA (1992). Neurotransmitter actions in the thalamus and cerebral cortex and their role in neuromodulation of thalamocortical activity. Prog Neurobiol 39: 337-388.

Miller CA (1995). Medications that may cause cognitive impairment in older adults. Geriatric Nursing 16: 47.

Morris RGM (1989). Synaptic plasticity and learning: selective impairment in rats and blockade of long term potentiation in vivo by the $N$-methyl-D-aspartate receptor antagonist AP5. J Neurosci 9: 3040-3057.

Morris RGM, Anderson E, Lynch GS, Baudry M (1986). Selective impairment of learning and blockade of long-term potentiation by an $N$-methyl-D-aspartate receptor antagonist, AP5. Nature 319: 774-776.

Nilsson OG, Strecker RE, Daszuta A, Bjorklund A (1988). Combined cholinergic and serotonergic denervation of the forebrain produces severe deficits in a spatial learning task in the rat. Brain Res 453: 235-246.

Richter-Levin G, Segal M (1989). Spatial performance is severely impaired in rats with combined reduction of serotonergic and cholinergic transmission. Brain Res 477: 404-407.

Rogers TV, Bowman CE (1990). Cognitive impairment associated with $\beta$-blockade in the elderly. Postgrad Med J 66: 1050-1052.

Sachdev PS, Sabaror T (1997). The effects of b-adrenoreceptor antagonists on a rat model of neuroleptic-induced akathisia. Psychiatr Res 72: 133-140.

Sara SJ, Dyon-Laurent C, Herve A (1995). Novelty seeking behavior in the rat is dependent upon the integrity of the noradrenergic system. Cog Brain Res 2: 181-187.

Saucier D, Cain DP (1995). Spatial learning without NMDA receptor-dependent long-term potentiation. Nature 378: 186189.

Saucier D, Hargreaves EL, Boon F, Vanderwolf CH, Cain DP (1996). Detailed behavioral analysis of water maze acquisition under systemic NMDA or muscarinic antagonism: nonspatial pretraining eliminates spatial learning deficits. Behav Neurosci 110: 103-116.

Schenk F, Morris RGM (1985). Dissociation between components of spatial memory in rats after recovery from the effects of retrohippocampal lesions. Exp Brain Res 58: 11-28.

Schiff AA, Saxey A (1984). Autoradiography of nadolol and propranolol in the rat. Xenobiotica 14: 687-691.

Sutherland RJ, Whishaw IQ, Regehr JC (1982). Cholinergic receptor blockade impairs spatial localization by use of distal cues in the rat. J Comp Physiol Psychol 96: 563-573.

Teri L, Larson EB, Reifler BV (1988). Behavioral disturbance in dementia of the Alzheimer's type. J Am Geriatr Soc 36: 1-6.

Vanderwolf CH (1987). Near-total loss of 'learning' and 'memory' as a result of combined cholinergic and serotonergic blockade in the rat. Behav Brain Res 23: 43-57.

Vanderwolf CH (1991). Anti-muscarinic drug effects in a swim-toplatform test: dose-response relations. Behav Brain Res 44: $217-$ 219.

Vanderwolf CH, Baker G (1996). The role of brain noradrenaline in cortical activation and behavior: a study of lesions of the locus coeruleus, medial thalamus and hippocampus-neocortex and of muscarinic blockade in the rat. Behav Brain Res 78: 225-234.

Westerlund A (1985). Central nervous system side-effects with hydrophilic and lipophilic $\beta$-blockers. Eur J Clin Pharmacol 28: 73-76.

Whishaw IQ (1989). Dissociating performance and learning deficits on spatial navigation tasks in rats subjected to cholinergic muscarinic blockade. Brain Res Bull 23: 347-358.

Whishaw IQ, Cassel JC, Jarrard LE (1995). Rats with fimbria-fornix lesions display a place response in a swimming pool: a dissociation between getting there and knowing where. $J$ Neurosci 15: 5779-5788.

Whishaw IQ, Jarrard LE (1995). Similarities $v s$ differences in place learning and circadian activity in rats after fimbria-fornix section or ibotenate removal of hippocampal cells. Hippocampus 5: 595-604.

Whishaw IQ, Jarrard LE (1996). Evidence for extrahippocampal involvement in place learning and hippocampal involvement in path integration. Hippocampus 6: 513-524.

Whishaw IQ, Tomie JA (1987). Cholinergic receptor blockade produces impairments in a sensorimotor subsystem for place navigation in the rat: evidence from sensory, motor, and acquisition tests in a swimming pool. Behav Neurosci 101: 603-616. 